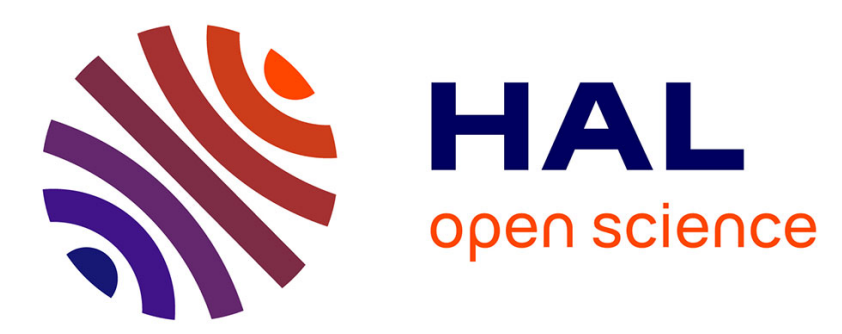

\title{
Influence of Recent Stratification Changes on ENSO Stability in a Conceptual Model of the Equatorial Pacific
}

Sulian Thual, Boris Dewitte, Soon-Il An, Serena Illig, Nadia Ayoub

\section{To cite this version:}

Sulian Thual, Boris Dewitte, Soon-Il An, Serena Illig, Nadia Ayoub. Influence of Recent Stratification Changes on ENSO Stability in a Conceptual Model of the Equatorial Pacific. Journal of Climate, 2012, 26, pp.4790-4802. 10.1175/JCLI-D-12-00363.1 . hal-00951084

\section{HAL Id: hal-00951084 https://hal.science/hal-00951084}

Submitted on 10 Jun 2014

HAL is a multi-disciplinary open access archive for the deposit and dissemination of scientific research documents, whether they are published or not. The documents may come from teaching and research institutions in France or abroad, or from public or private research centers.
L'archive ouverte pluridisciplinaire HAL, est destinée au dépôt et à la diffusion de documents scientifiques de niveau recherche, publiés ou non, émanant des établissements d'enseignement et de recherche français ou étrangers, des laboratoires publics ou privés. 


\title{
Influence of Recent Stratification Changes on ENSO Stability in a Conceptual Model of the Equatorial Pacific
}

\author{
Sulian Thual AND Boris DewitTe \\ Laboratoire d'Etudes en Géophysique et Océanographie Spatiales, Toulouse, France \\ SOON-IL AN \\ Department of Atmospheric Sciences, Yonsei University, Seoul, South Korea \\ Serena Illig AND NADia Ayoub \\ Laboratoire d'Etudes en Géophysique et Océanographie Spatiales, Toulouse, France
}

(Manuscript received 21 June 2012, in final form 13 November 2012)

\begin{abstract}
Changes in the mean circulation of the equatorial Pacific Ocean partly control the strong decadal modulation of El Niño-Southern Oscillation (ENSO). This relationship is considered from the linear stability of a conceptual recharge/discharge model with parameters tuned from the observed mean state. Whereas decadal changes in the mean thermocline depth alone are usually considered in conceptual ENSO models, here focus is given to decadal changes in the mean stratification of the entire upper ocean (e.g., the mean thermocline depth, intensity, and thickness). Those stratification changes modify the projection of wind stress forcing momentum onto the gravest ocean baroclinic modes. Their influence on the simulated frequency and growth rate is comparable in intensity to the one of usual thermodynamic and atmospheric feedbacks, while they have here a secondary effect on the spatial structure and propagation of SST anomalies. This sensitivity is evidenced in particular for the climate shift of the 1970s in the Simple Ocean Data Assimilation (SODA) dataset, as well as in a preindustrial simulation of the Geophysical Fluid Dynamics Laboratory (GFDL) model showing stratification changes similar to the ones after 2000. Despite limitations of the linear stability approach, conclusions on the sensitivity to stratification may be extended to interpret the modulation and diversity of ENSO in observations and in general circulation models.
\end{abstract}

\section{Introduction}

El Niño-Southern Oscillation (ENSO) is a natural mode of variability of the equatorial Pacific that experiences a strong modulation of its characteristics at decadal to interdecadal time scales. For example, after the 1970s, ENSO characteristics were modified toward less frequent and stronger events, with additionally a tendency toward more eastward propagating SST anomalies (Wang and Wang 1996; An et al. 2006). Characteristics of ENSO have also changed dramatically over the recent decades, with an increased occurrence of central Pacific (CP) El

Corresponding author address: Sulian Thual, Laboratoire d'Etude en Géophysique et Océanographie Spatiale, 14 av Edouard Belin, 31400 Toulouse, France.

E-mail: sulian.thual@gmail.com
Niño events characterized by maximal SST anomalies in the central Pacific, different from eastern Pacific (EP) El Niño events (Ashok et al. 2007; Kug et al. 2009; Lee and McPhaden 2010). In addition, the amplitude and phase lag of the thermocline recharge/discharge process (Jin 1997a,b) has weakened after 2000, suggesting changes in the mechanisms and predictability of ENSO (McPhaden 2012). The modulation of ENSO characteristics remains a critical issue for the scientific community, as it continuously challenges established theoretical understanding of ENSO properties as well as the reliability of forecasting systems (Kirtman and Schopf 1998; Neelin et al. 1998; McPhaden 2012).

One unresolved issue regarding ENSO modulation is its relation to changes in the mean circulation of the equatorial Pacific. Figure 1a shows changes in mean temperatures at the equator after the climate shift of the 

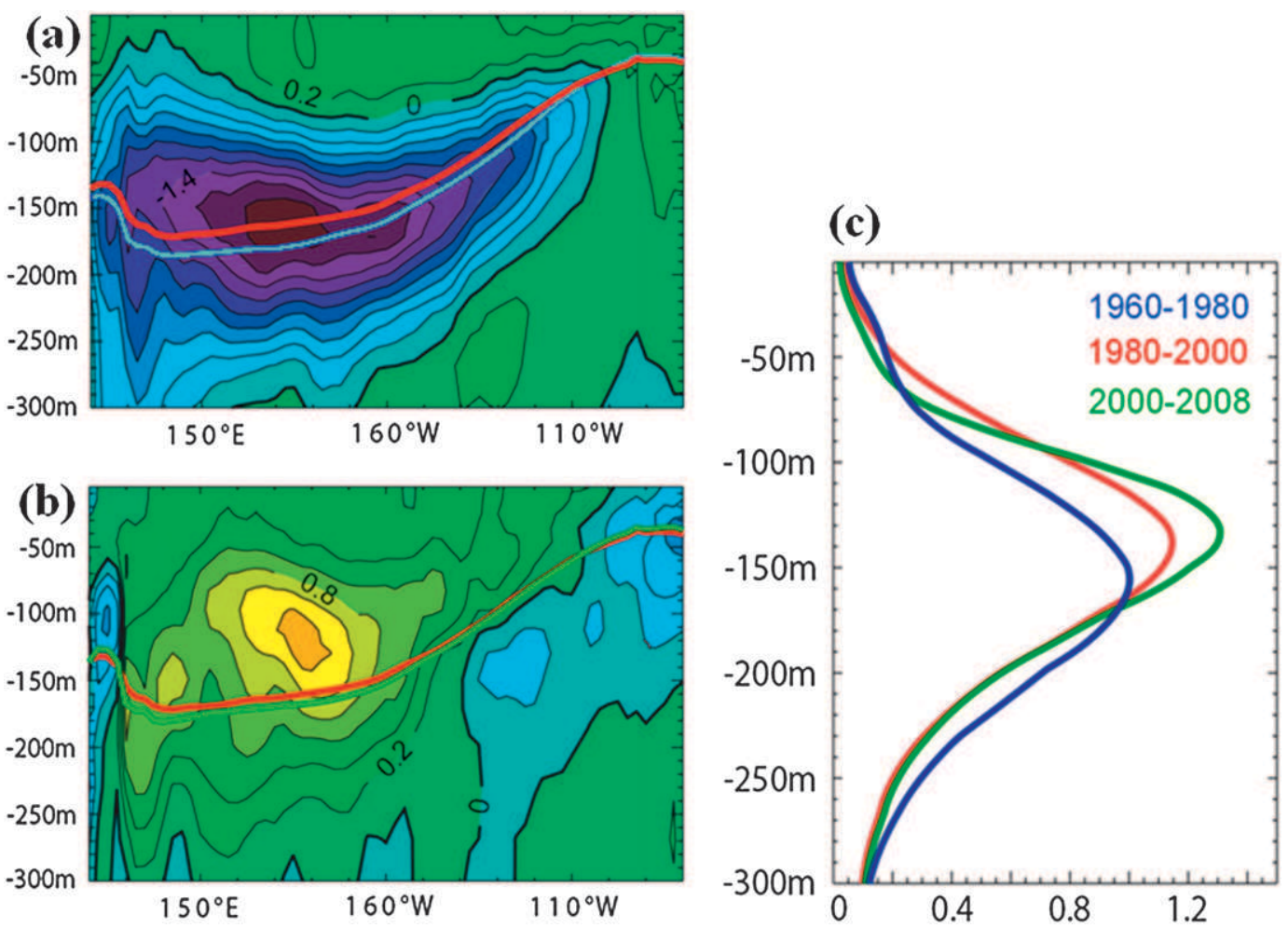

FIG. 1. Mean temperature difference (a) 1980-2000 minus 1960-80 and (b) 2000-08 minus 1980-2000, in the SODA reanalysis, at the equator and as a function of longitude and depth. Contour interval (CI) is $0.2^{\circ} \mathrm{C}$. The mean $20^{\circ} \mathrm{C}$ isotherm (D20) is superimposed for 1960-80 (blue), 1980-2000 (red), and 2000-08 (green). (c) The Brunt-Väisälä frequency $N^{2}\left(\mathrm{~min}^{-2}\right.$; averaged within $\left.170^{\circ} \mathrm{E}-170^{\circ} \mathrm{W}\right)$ as a function of depth for each period. Changes are significant at the $99 \%$ level from a Student's $t$ test assuming unequal variance, except in the first $50 \mathrm{~m}$ (at depth $110 \mathrm{~m}$ ) for the climate shift of the 1970s (the mean state change of 2000).

1970s, evidencing a shallowing of the equatorial-mean thermocline as well as a vertical stratification increase, with a $0.2^{\circ} \mathrm{C}$ warming at the surface and a $1.8^{\circ} \mathrm{C}$ cooling in subsurface temperatures (Moon et al. 2004; Dewitte et al. 2009). Despite the relatively short record, changes in the mean state are also observed after 2000 (Fig. 1b), with a strengthening of the equatorial Pacific warm pool associated with a slight deepening of the equatorialmean thermocline, a $0.2^{\circ} \mathrm{C}$ warming in surface, and a $1.2^{\circ} \mathrm{C}$ warming in subsurface. One established understanding of ENSO is that it is a near-neutral mode of oscillation that arises from a destabilization of the equatorial Pacific-mean circulation rather than from external forcing alone (Neelin et al. 1998; Philander and Fedorov 2003). Therefore, although ENSO modulation in observations and simulations is related to many aspects of the climatic system, such as decadal modes of variability, ENSO asymmetry, seasonal cycle interaction, or random fluctuations (Jin et al. 1994; Jin 2001; Rodgers et al. 2004; Seager et al. 2004), some of its characteristics have been shown to be related to changes in the destabilized background mean state
(Fedorov and Philander 2001; An and Jin 2001; Sun and Zhang 2006; Choi et al. 2011). This relation is also of particular interest in a context of global warming, for which climate projections by coupled general circulation models (CGCMs) show an overall agreement on a modification toward a warmer mean state of the equatorial Pacific, but an overall disagreement on an increase or decrease of ENSO activity (Guilyardi et al. 2009). Those models indeed simulate their own mean state with a large and complex set of compensating ENSO feedbacks, among which a critical challenge is to identify which feedbacks are most influential on the simulated ENSO modulation (Collins et al. 2010; Kim and Jin 2011; Belmadani et al. 2010).

Among the various mean state feedbacks (i.e., parameters) that may be influential on ENSO modulation, this article focuses in particular on the upper-ocean stratification of the equatorial Pacific. Figure 1c shows changes in the Brunt-Väisälä frequency profile $N^{2}$, (which is proportional to the vertical gradient of density, i.e., the buoyancy or stratification) for the climate shift of the 1970s and for the mean conditions after 2000. It 
TABLE 1. Correlation, among the panel of 15 CGCM simulations, between the 20-yr running std dev of Niño-3 SST anomalies [i.e., the index N3Var as in Rodgers et al. (2004)], and the 20-yr running mean of $P_{1}, P_{2}, P_{3}$ (averaged within $170^{\circ} \mathrm{E}-170^{\circ} \mathrm{W}$ ), and of D20 (mean thermocline depth in area Niño-4). Models are ordered by decreasing correlation of $P_{2}$ and $P_{3}$. All time series were detrended.

\begin{tabular}{|c|c|c|c|c|c|}
\hline \multicolumn{2}{|l|}{ Model } & \multicolumn{3}{|c|}{$P_{n}$ coefficients } & \multirow[b]{2}{*}{$\mathrm{D} 20$} \\
\hline Expansion & Acronym & $P_{1}$ & $P_{2}$ & $P_{3}$ & \\
\hline $\begin{array}{l}\text { ECHAM (Meteorological Institute of the University of Bonn) } \\
\text { and the global Hamburg Ocean Primitive Equation }\end{array}$ & ECHO-G & -0.53 & 0.81 & 0.82 & -0.54 \\
\hline $\begin{array}{l}\text { Centre National de Recherches Météorologiques (CNRM) } \\
\text { Coupled Global Climate Model, version } 3\end{array}$ & CNRM-CM3 & -0.49 & 0.78 & 0.75 & -0.75 \\
\hline $\begin{array}{l}\text { Geophysical Fluid Dynamics Laboratory (GFDL) Climate } \\
\text { Model, version } 2.1\end{array}$ & GFDL CM2.1 & -0.47 & 0.60 & 0.72 & -0.57 \\
\hline ECHAM5 (Max Planck Institute) & ECHAM5 & 0.46 & 0.62 & 0.62 & -0.70 \\
\hline $\begin{array}{l}\text { Institute of Numerical Mathematics (INM) Coupled Model, } \\
\text { version 3.0 }\end{array}$ & INM-CM3.0 & 0.10 & 0.62 & 0.61 & -0.71 \\
\hline $\begin{array}{l}\text { Commonwealth Scientific and Industrial Research Organisation } \\
\text { (CSIRO) Mark, version } 3.0\end{array}$ & CSIRO Mk3.0 & -0.29 & 0.57 & 0.64 & -0.36 \\
\hline GFDL Climate Model, version 2.0 & GFDL CM2.0 & -0.40 & 0.46 & 0.73 & -0.33 \\
\hline CSIRO Mark, version 3.5 & CSIRO Mk3.5 & -0.32 & 0.54 & 0.47 & -0.21 \\
\hline Hadley Centre Coupled Model, version 3 & HadCM3 & -0.19 & 0.46 & 0.53 & -0.44 \\
\hline Flexible Global Ocean-Atmosphere-Land System Model, run 1 & FGOALSrun1 & -0.03 & 0.64 & 0.28 & -0.39 \\
\hline $\begin{array}{l}\text { Meteorological Research Institute (MRI) Coupled General } \\
\text { Circulation Model, version 2.3.2a }\end{array}$ & MRI CGCM2.3.2a & -0.25 & 0.36 & 0.32 & -0.54 \\
\hline $\begin{array}{l}\text { National Center for Atmospheric Research (NCAR) Community } \\
\text { Climate System Model, version } 3.0\end{array}$ & CCSM3.0 & -0.52 & 0.18 & 0.42 & 0.00 \\
\hline L'Institut Pierre-Simon Laplace (IPSL) Coupled Model, version 4 & IPSL-CM4 & -0.21 & 0.03 & 0.52 & 0.28 \\
\hline Istituto Nazionale di Geofisica e Vulcanologia (INGV) ECHAM4 & ECHAM4 & 0.18 & -0.03 & 0.30 & 0.07 \\
\hline Hadley Centre Global Environmental Model, version 1 & HadGEM1 & -0.24 & -0.20 & 0.02 & 0.22 \\
\hline
\end{tabular}

evidences changes in both the thermocline depth (defined where $N^{2}$ is maximal) and "sharpness" (i.e., amplitude and vertical extension of $N^{2}$ ). To account for the sensitivity of ENSO to this particular parameter, a refined representation of the ocean vertical structure is necessary. This may be achieved by considering the gravest baroclinic modes of the continuously stratified ocean (Dewitte 2000; Moon et al. 2004; Dewitte et al. 2007, 2009; Thual et al. 2011, hereafter TD2011). One critical aspect that will be considered here is how decadal changes in stratification modify the projection of wind stress forcing onto the gravest ocean baroclinic modes.

This sensitivity to stratification is accounted for in the recharge/discharge conceptual model from TD2011 (herein called the TD model). In this anomaly model, the mean state is prescribed and controls the linear stability of the so-called ENSO mode (i.e., its oscillation frequency, growth rate, and spatial structure). Such types of reduced-to-intermediate models that focus on fundamental oscillation mechanisms have been successful in interpreting several aspects of ENSO modulation (An and Jin 2000; An and Wang 2000; Fedorov and Philander 2001; An and Jin 2001). They have also allowed for synthetic intercomparison of most influential ENSO feedbacks in observations and CGCM simulations with, for example, the Bjerknes index (Jin et al. 2006; Kim and Jin 2011) that is built from the linear stability of the recharge/discharge model of Jin (1997a). In line with those former studies, the TD model has been shown to reproduce the linear stability, driving mechanisms, and main parameter sensitivity of ENSO (TD2011). Its peculiarity, however, is its particular sensitivity to upper-ocean stratification, which is not taken into account in former reduced models with a two-layer ocean.

This article extends the study of TD2011, where here the sensitivity of the simulated ENSO stability to upper-ocean stratification is assessed for realistic cases of mean state changes such as the climate shift of the 1970s in the Simple Ocean Data Assimilation (SODA) reanalysis (Carton and Giese 2008), as well as in a preindustrial simulation of the Geophysical Fluid Dynamics Laboratory (GFDL) Climate Model, version 2.1 (CM2.1) (Wittenberg et al. 2006; note that all model names are expanded in Table 1), showing stratification changes similar to the ones after 2000. The article is organized as follows: Section 2 recalls the main characteristics of the TD model. Section 3 documents mean state and stability changes during the climate shift of the 1970s. Section 4 documents mean state and stability changes in the GFDL model, with considerations also extended to a panel of other preindustrial CGCM simulations. Section 5 is a discussion followed by concluding remarks. 


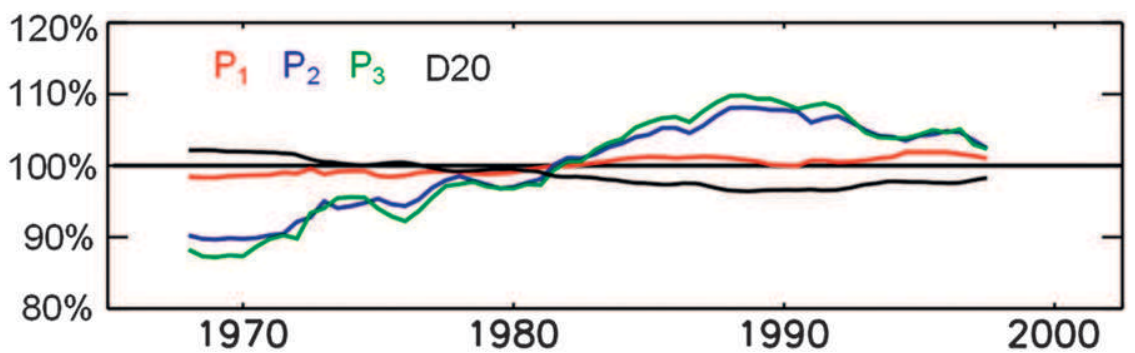

FIG. 2. Running mean (20 yr) of projection coefficients $P_{1}$ (red), $P_{2}$ (blue), and $P_{3}$ (green), and mean thermocline depth D20 (black; averaged in area Niño-4), in percent of their mean value over 1958-2008 in the SODA reanalysis.

\section{Method}

In this section, we briefly recall the main characteristics of the TD model, which is used for linear stability analysis through tuning of its parameters from the observed mean state. The reader is also invited to refer to TD2011 for more details. We also describe briefly the preindustrial datasets that will be used in sections 3 and 4.

\section{a. The TD model}

The TD model is a linear model of the equatorial Pacific that considers the evolution of anomalies with respect to a prescribed mean state. It solves the first equatorial waves for the three gravest baroclinic modes of a continuously stratified ocean (derived from the Boussinesq approximation). This reads, for each baroclinic mode $n(n=1,2,3)$, as follows:

$$
\begin{aligned}
\left(\partial_{t}+\varepsilon_{n}\right) K_{n}+c_{n} \partial_{x} K_{n}= & \frac{P_{n} \int_{-\infty}^{+\infty} \tau_{x} \psi_{0} d y}{2 \rho_{o} L_{n} c_{n}} \text { and } \\
\left(\partial_{t}+\varepsilon_{n}\right) R_{n}-\left(c_{n} / 3\right) \partial_{x} R_{n} & =\frac{P_{n} \int_{-\infty}^{+\infty}\left[\left(\tau_{x} \psi_{2} / \sqrt{2}\right)-\tau_{x} \psi_{0}\right] d y}{3 \rho_{o} L_{n} c_{n}},
\end{aligned}
$$

with conditions of reflection at the eastern and western boundaries (not shown). Here, $\tau_{x}$ is zonal wind stress forcing anomalies and $\rho_{o}$ is ocean-mean density. For each baroclinic mode, $K_{n}$ and $R_{n}$ are the amplitude of the Kelvin and first Rossby wave, respectively, $\varepsilon_{n}$ is a dissipation rate, and $c_{n}$ is the phase speed of Kelvin waves. Also, $\psi_{0}=\phi_{0}\left(y / L_{n}\right)$ and $\psi_{2}=\phi_{2}\left(y / L_{n}\right)$, where $\phi_{0}$ and $\phi_{2}$ are the zeroth- and second-order Hermite functions, respectively, $L_{n}=\sqrt{c_{n} / \beta}$ is the ocean Rossby radius, and $\beta$ is the beta-plane parameter. The contributions from all equatorial waves and baroclinic modes are solved and then summed to reconstruct both thermocline and zonal current anomalies (see TD2011).
The efficiency of transfer of mechanical work from wind stress forcing to ocean motion is set by projection coefficients $P_{n}$, which are computed for the three gravest ocean baroclinic modes $(n=1,2,3)$ as

$$
P_{n}=\frac{1}{\int_{H}^{0} F_{n}^{2} d z},
$$

where $H$ is the ocean bottom depth and $F_{n}$ is the vertical function associated to the baroclinic mode $n$. The vertical functions $F_{n}$ (and therefore the $P_{n}$ coefficients) are computed from the Brunt-Väisälä frequency $N^{2}$ averaged over the central Pacific $\left(170^{\circ} \mathrm{E}-170^{\circ} \mathrm{W}\right)$ where decadal variability is most marked (Dewitte 2000; see also Fig. 1). Figure 2 shows variations in the $P_{n}$ coefficients estimated from the SODA reanalysis, with marked decadal variations over the recent decades that reach up to $20 \%$ of their nominal values and that are anticorrelated with variations in the central Pacific-mean thermocline depth (D20). Noteworthy changes in $P_{1}$ are weak while $P_{2}$ and $P_{3}$ capture the essential stratification variability at decadal time scales (Dewitte et al. 2007).

In addition, the TD model considers dominant processes of oceanic mixed layer thermodynamics that control the evolution of sea surface temperature (SST) anomalies in an equatorial strip, consisting of a zonal advective feedback $F_{U}$, thermocline feedback $F_{H}$, and damping feedback $F_{W}$. It further considers coupling to a statistical atmosphere that relates zonal wind stress anomalies to SST anomalies, with two responses $\mu_{1}$ and $\mu_{2}$ built from the two first modes of a singular value decomposition ( $66 \%$ and $15 \%$ of covariance, respectively). This reads

$$
\begin{aligned}
\partial_{t} T & =F_{W} T+F_{H} h+F_{U} u \text { and } \\
\tau_{x} & =\mu_{1}(T)+\mu_{2}(T),
\end{aligned}
$$

where $T, h$, and $u$ are anomalies of SST, thermocline depth, and zonal current, respectively. 


\section{b. SODA and CGCMs}

In this article, TD model parameters are tuned from the SODA reanalysis, version 1.2.6 (Carton and Giese 2008), and from various CGCM simulations derived from the archive of phase 3 of the Coupled Model Intercomparison Project (CMIP3). For the CGCMs, preindustrial simulations are used with the concentration of greenhouse gases fixed to 1850 estimates, which makes it appropriate for studying the physical mechanisms of natural climate variability. The simulations are listed in Table 1. They correspond to the models selected in Belmadani et al. (2010), except for BCCR-BCM2.0. They span around 150 years except for GFDL CM2.1 for which we consider a longer preindustrial simulation of 300 years (Wittenberg et al. 2006). The GFDL simulation has been extensively analyzed in recent studies (Kug et al. 2010; Choi et al. 2011; Dewitte et al. 2012) because of the ability of the GFDL model to simulate an alternation of periods with increased occurrence of central or eastern Pacific El Niño events.

\section{Climate shift of the $1970 \mathrm{~s}$}

In this section, we compute the stability of the TD model whose parameters are tuned from the varying mean state of the SODA reanalysis spanning 1958-2008 (Carton and Giese 2008). Only a reduced set of the TD model parameters are tuned from this varying mean state, while the remaining parameters are estimated over the entire SODA record as in TD2011. For the ocean, only the $P_{n}$ coefficients are tuned because they show the most marked decadal variations among parameters from Eqs. (1) and (2) (Dewitte et al. 2007). For mixed layer thermodynamics [Eq. (4)], the zonal advective feedback (i.e., $F_{U}$ ) is scaled by the mean SST difference between $150^{\circ}$ and $90^{\circ} \mathrm{W}$ and between $120^{\circ} \mathrm{E}$ and $180^{\circ}$ (An and Jin 2000). The thermocline feedback $F_{H}$ is scaled by the mean difference between SST and subsurface temperature (from 0 to $100 \mathrm{~m}$ ) over $150^{\circ}-$ $90^{\circ} \mathrm{W}$ (An et al. 2008). The damping feedback (i.e., $F_{W}$ ) is scaled by the intensity of mean zonal wind stress over $150^{\circ}-90^{\circ} \mathrm{W}$ (which controls the mean upwelling intensity). Finally, the statistical atmosphere responses (i.e., $\mu_{1}$ and $\mu_{2}$ ) [Eq. (5)] are scaled by the correlation of time series from their respective singular value decomposition (SVD) modes, whereas those SVD modes are computed over the entire SODA record.

The TD model is linear with respect to initial conditions, and, for a given set of model parameters, its eigenmodes (eigenvectors and eigenvalues) are computed from a linear stability analysis (see, e.g., Fig 2 of TD2011). As in TD2011, the so-called ENSO mode is defined as the (a)

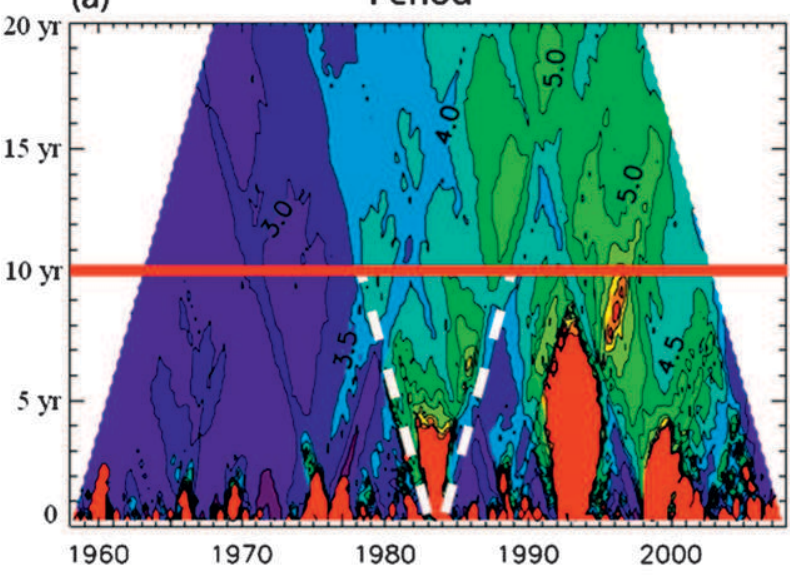

(b) Growth rate

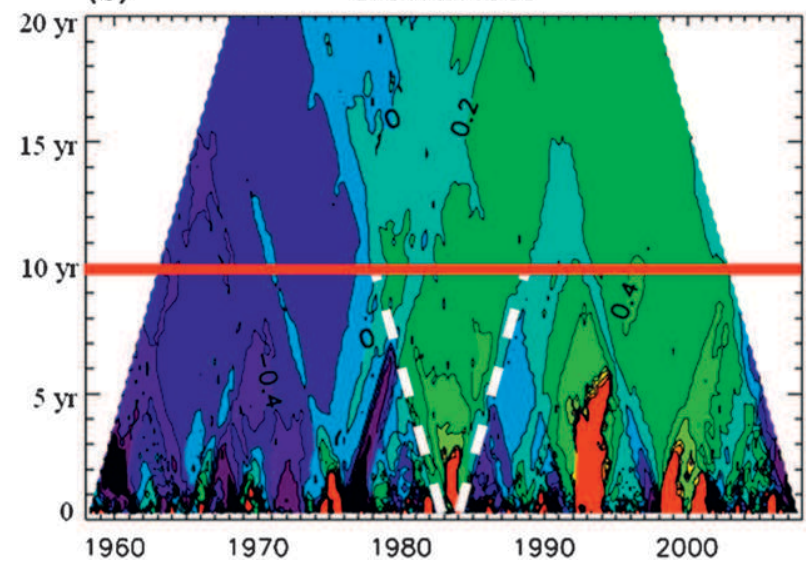

FIG. 3. (a) Period (yr) and (b) growth rate $\left(\mathrm{yr}^{-1}\right)$ of the simulated ENSO mode, as a function of the window center ( $x$ axis; yr) and window length ( $y$ axis; yr) used for the tuning of the TD model parameters from the SODA reanalysis. For the period (growth rate), CI increases from blue to red with a step of 0.5 years $\left(0.2 \mathrm{yr}^{-1}\right)$. Values $>7 \mathrm{yr}\left(1.2 \mathrm{yr}^{-1}\right)$ are not contoured. Stability changes for short window intervals ( $<10 \mathrm{yr}$; cf. red horizontal line) are spurious and may result from specific El Niño events (e.g., white dashed lines for El Niño 1983).

eigenmode with maximum growth rate. The model also exhibits various secondary eigenmodes, but they remain here significantly more damped (with growth rate around $-1.5 \mathrm{yr}^{-1}$ ) and are therefore not considered.

Figure 3 shows the linear stability of the simulated ENSO mode, namely its period and growth rate, as a function of the position and length of the window interval used to tune model parameters (i.e., $P_{n}, F_{U}, F_{H}$, $F_{W}, \mu_{1}$, and $\mu_{2}$ ) from the varying mean state of the SODA reanalysis. This tests the significance of mean state and stability changes with respect to shorter-term climatic fluctuations. Specific events of interannual variability (e.g., El Niño in 1983, 1992, and 1998) may result in spurious stability shifts for short window 


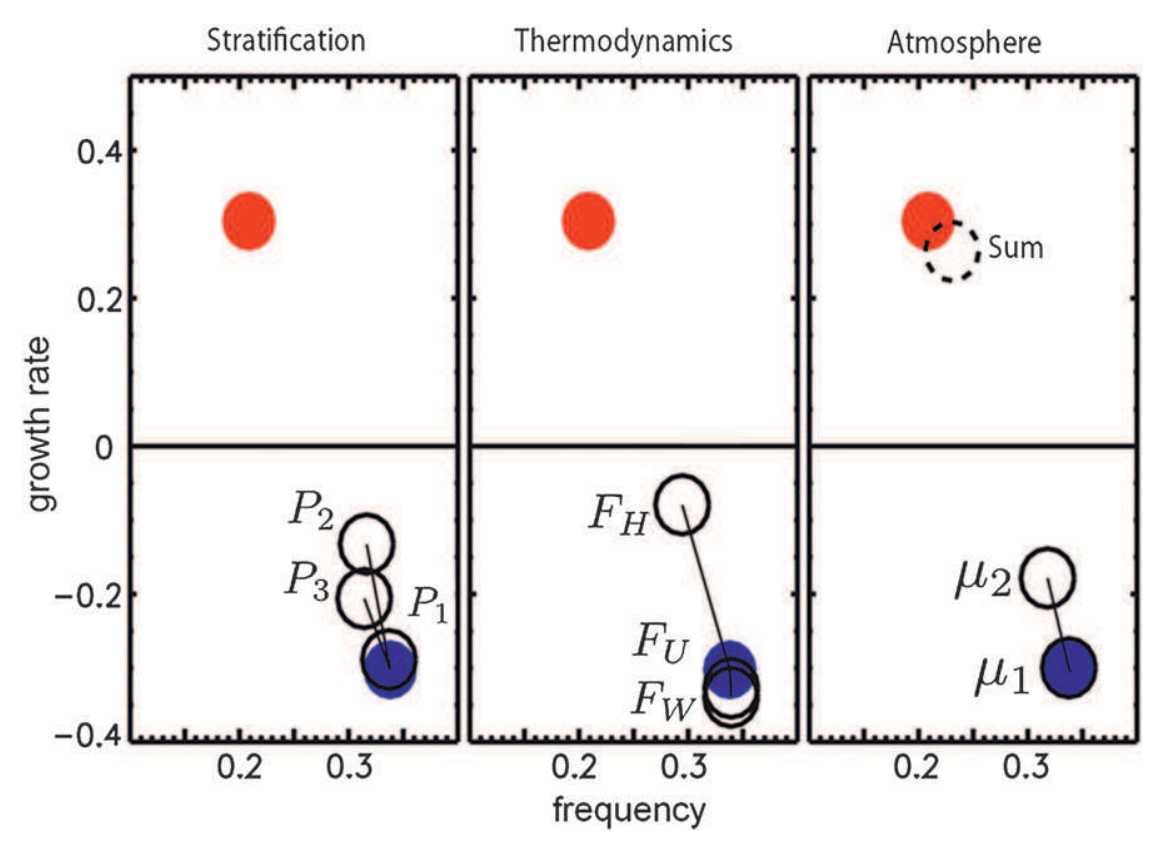

FIG. 4. Frequency $\left(\mathrm{yr}^{-1}\right)$ and growth rate $\left(\mathrm{yr}^{-1}\right)$ of the TD model-simulated ENSO mode, for the climate shift of the 1970s. Stability values are shown for tuning the SODA record using either the window interval 1960-80 (blue) or 1980-2000 (red). The open black circles are obtained by individually tuning each parameter from 1980 to 2000 with other parameters tuned from 1960 to 1980 . The "sum point" (black open dashed circle) sums all stability changes from individual parameter changes.

intervals, as seen on associated diagonal rays (see, e.g., the white dashed lines for the effect of the 1983 El Niño). Stability changes for window intervals shorter than 10 years are obviously spurious (cf. red horizontal line) and are shown here only for illustration purpose, while stability changes become more clearly separable from interannual variability for longer window intervals ( $\sim 20 \mathrm{yr})$. This notably reveals the difficulty, because of the short record, to find evidence of changes in stability after 2000, despite the observations that some ENSO properties have been changing, especially with an increased occurrence of central Pacific El Niño events (Lee and McPhaden 2010; McPhaden 2012). The relation between mean circulation and the occurrence of the central Pacific El Niño will be overlooked in a following section based on the analysis of a preindustrial CGCM simulation that spans a sufficiently long period to reach statistical confidence. Here the main feature of the SODA record is rather the climate shift of the 1970s, which consists of an increase in period and growth rate after 1980 that is separable from interannual fluctuations for a window interval around 20 years.

Changes in stability for the climate shift of the 1970s are further shown in Fig. 4. Here the TD model parameters are tuned from either the window interval 1960-80 (blue) or 1980-2000 (red). Despite the TD model's simplicity, the simulated increase in frequency and growth rate after 1980 is qualitatively consistent with the decrease (increase) in ENSO occurrence (amplitude) from the observed record (Wang and Wang 1996; An and Jin 2000). This corresponds to modified scaling of model parameters after 1980 , on $P_{1}(+3 \%), P_{2}$ $(+25 \%)$, and $P_{3}(+18 \%)$ for the upper-ocean stratification; $F_{U}(-5 \%), F_{H}(+18 \%)$, and $F_{W}(-11 \%)$ for mixedlayer thermodynamics; and $\mu_{1}(+1 \%)$ and $\mu_{2}(+66 \%)$ for the statistical atmosphere. Noteworthy changes are weak for $P_{1}$ but marked for $P_{2}$ and $P_{3}$ (see also Fig. 2). Changes in parameters of mixed layer thermodynamics are in qualitative agreement with An and Jin (2000), while the increase in atmospheric response $\mu_{2}$ somewhat reflects the eastward shift of the zonal wind stress with respect to the SST anomalies after 1980 (An and Wang 2000).

As an attempt to quantify the sensitivity to each model parameter, the model stability is recomputed with tuning from 1960 to 1980 except for a single parameter tuned from 1980 to 2000 (black open circles in Fig. 4). This reveals the contribution of each parameter to the total change in stability and its relative influence on ENSO stability. The influence of $P_{1}$, for example, is much weaker than that of $P_{2}$ and $P_{3}$. The influence of mixed layer thermodynamic processes has already been depicted in An and Jin (2000), though this study did not disentangle the relative influence of the thermocline and zonal advective feedbacks. Here it appears that the 
(a) SST

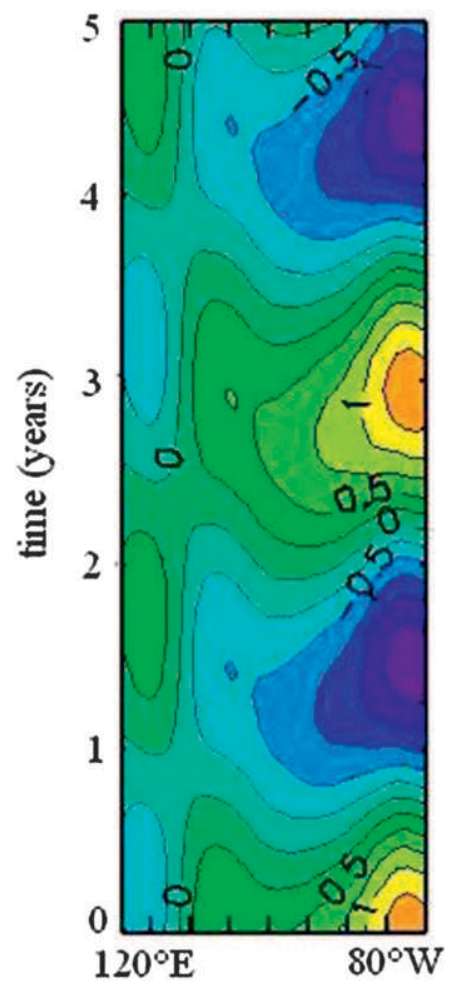

(b) Thermocline

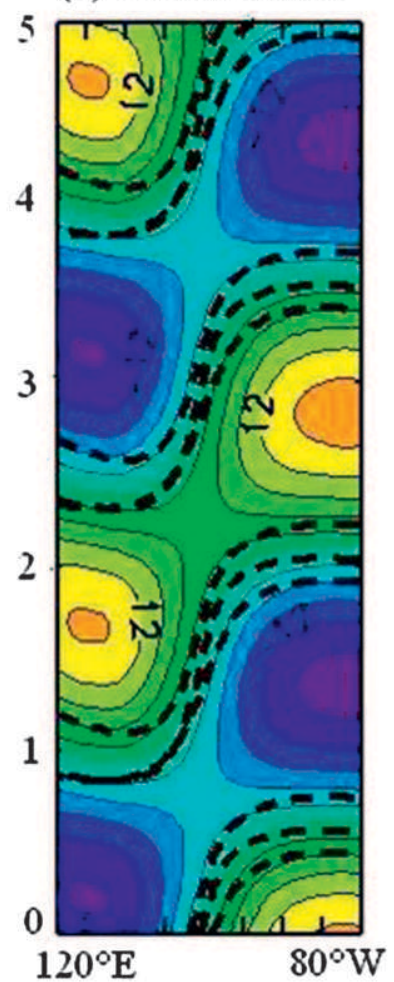

(c) SST

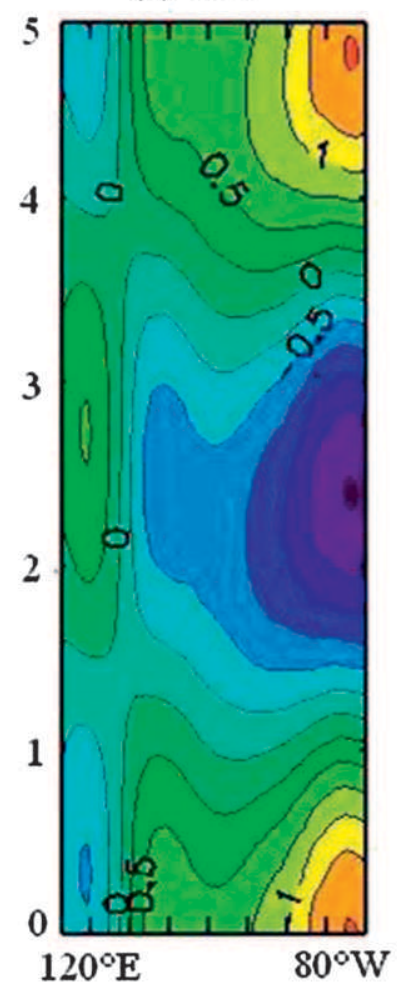

(d) Thermocline

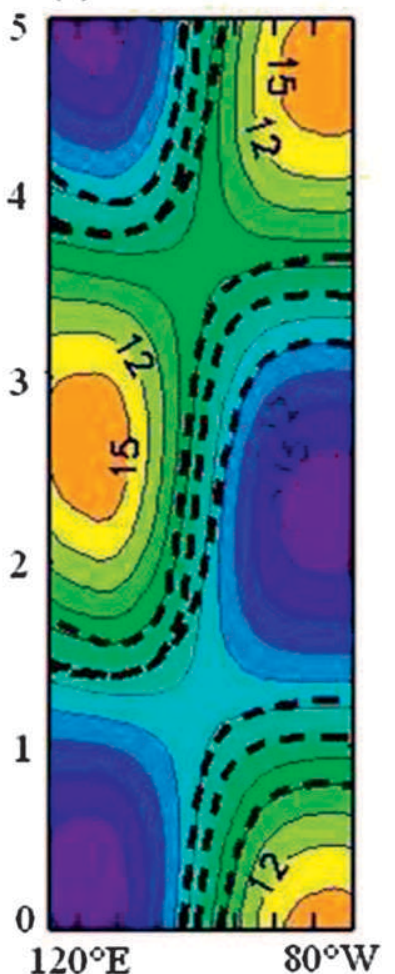

FIG. 5. (a) SST and (b) thermocline depth anomalies, as a function of longitude and time, reconstructed from the ENSO mode eigenvector of 1960-80 with growth rate omitted. (c) SST and (d) thermocline depth anomalies from the ENSO mode eigenvector of 19802000. CI is $0.25^{\circ} \mathrm{C} \mathrm{(3} \mathrm{m)} \mathrm{for} \mathrm{SST} \mathrm{(thermocline} \mathrm{depth)} \mathrm{anomalies.} \mathrm{Black} \mathrm{dashed} \mathrm{lines} \mathrm{in} \mathrm{(b)} \mathrm{and} \mathrm{(d)} \mathrm{are} \mathrm{the} \mathrm{zero} \mathrm{contours} \mathrm{for} \mathrm{the} \mathrm{contribution}$ of the three gravest baroclinic modes $(n=1,2,3)$ to thermocline depth anomalies.

thermocline feedback $F_{H}$ is most influential, at least in the TD model. In addition, the atmospheric response (i.e., $\mu_{2}$ ) leads to moderate changes in stability. As a consistency check, we also compute the sum of each stability change from single parameter change (black open dashed circle). It approximately matches the stability from 1980 to 2000 (red), which validates our hypothesis that stability changes depend linearly on parameter changes. In conclusion, a main result from Fig. 4 is that the influence of stratification parameters (i.e., $P_{2}$ and $P_{3}$ ) onto ENSO stability is comparable in magnitude to that of mixed layer thermodynamics and atmosphere parameters. This indicates that sensitivity to stratification parameters (i.e., $P_{n}$ ) also needs to be taken into account.

Figure 5 shows the spatial structure of SST and thermocline depth anomalies reconstructed from the eigenvectors of ENSO modes of Fig. 4 (where the tuning of the TD model is either from the 1960-80 or 1980-2000 periods). They remain similar after 1980 except for the increased period of oscillation, and there is in particular no significant change in SST propagation as in the observed record (An et al. 2006). This limitation may arise from the model statistical atmosphere (built from two
SVD modes) that may constrain the location of the center of action of wind stress anomalies too much, and therefore limit the zonal propagation of coupled instabilities. Such a limitation could be overcome by further considering higher SVD modes, or alternatively a dynamical atmosphere (Neelin et al. 1998; Fedorov and Philander 2001) but is out of the scope of this article. Despite this, the TD model simulates a spatially fixed recharge/discharge process (Jin 1997a,b) that is qualitatively representative of ENSO. This is seen from maximal SST anomalies in the eastern Pacific in phase with a see-saw pattern of thermocline depth anomalies and out of phase with the equatorial heat content as seen from the thermocline depth anomalies having the same sign all along the equator. We also note from the zero contours (black dashed lines) that the contribution of each baroclinic mode to thermocline depth anomalies follows a similar recharge/discharge process, although with a slight delay on higher baroclinic modes.

The increased stratification after 1980 modifies the frequency and growth rate of the simulated ENSO mode (cf. Fig. 4) but has little impact on its spatial structure (cf. Fig. 5). According to Eqs. (1) and (2), it appears that the 
(a)
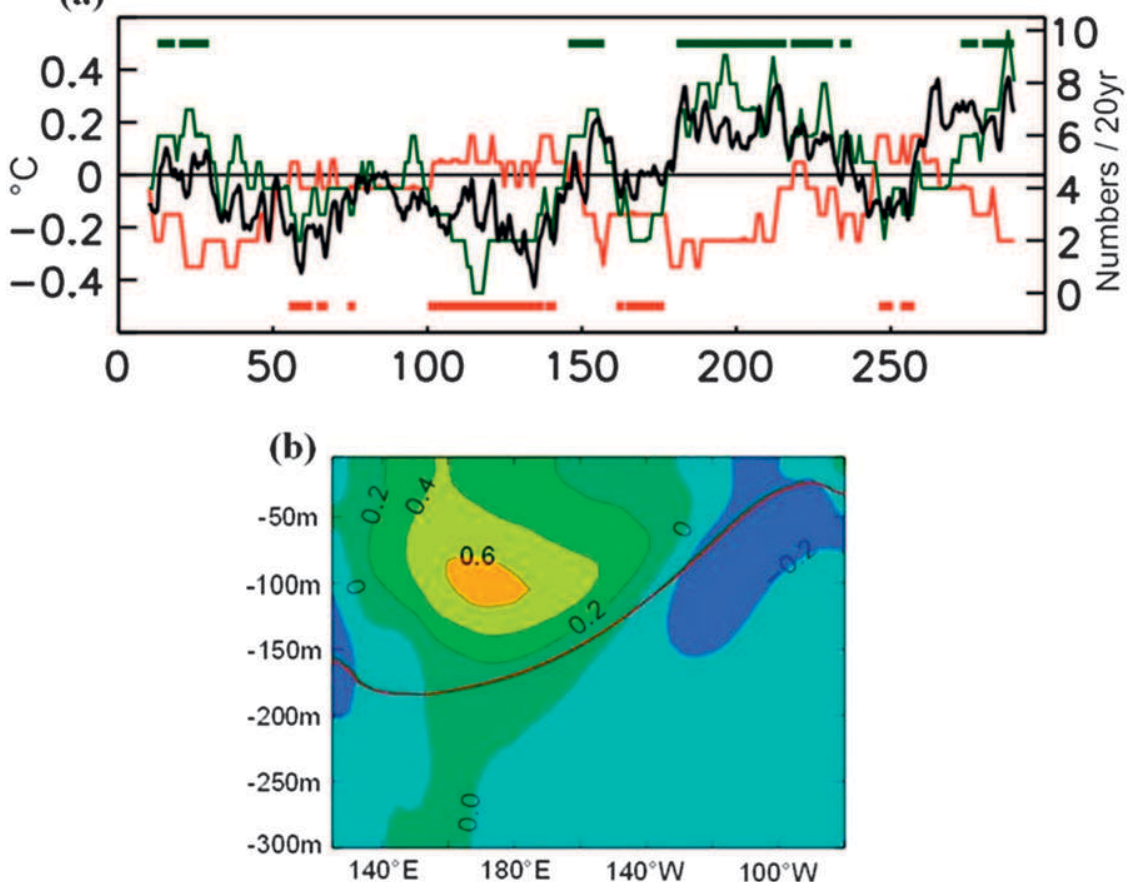

FIG. 6. (a) Decadal variability and decadal modulation of ENSO in the GFDL CM2.1 simulation, as a function of time from the beginning of the simulation ( $x$ axis; yr). Green (red) thin line indicates 20-yr frequency of CP (EP) events, in numbers by 20 -yr increments (right $y$ axis). Black line indicates 20 -yr running mean of SST averaged within Niño4m region $\left({ }^{\circ} \mathrm{C}\right.$; left $y$ axis). Green (red) horizontal thick lines in the top (bottom) indicate period intervals used to compute the warm (cold) mean state. Adapted from Kug et al. (2010) and Choi et al. (2011). (b) Temperature differences at the equator between the warm and cold mean states, as a function of longitude and depth. $\mathrm{CI}$ is $0.2^{\circ} \mathrm{C}$ and the $20^{\circ} \mathrm{C}$ isotherm depth is drawn for the cold mean state (red, almost identical for the warm mean state).

increased stratification (i.e., $P_{n}$ coefficients) enhances the intensity of the thermocline response, because for the same atmospheric forcing the ocean baroclinic modes trap more momentum (Dewitte 2000). This somewhat increases the intensity of ocean-atmosphere coupling and as such modifies the simulated growth rate and frequency (Jin 1997a; An and Jin 2001). There is also a slight decrease in the simulated amplitude of heat content (warm water volume) anomalies after 1980, which is caused by a slight increase in the phase between heat content and SST anomalies (not shown). A similar result is found by TD2011 (their Fig. 10) when directly increasing the intensity of ocean-atmosphere coupling, which is best interpreted in terms of a change in the regime of oscillation rather than only in the direct thermocline response to wind stress forcing.

\section{Decadal changes in stratification and ENSO stability}

Because the SODA reanalysis only allows us to address the sensitivity to stratification during the climate shift of the 1970s, in this section we also analyze various CGCM simulations that grasp some aspects of ENSO modulation (Lin 2007). As a first step, we perform a stability analysis similar to the one of the previous section with the TD model tuned from the mean state outputs of a preindustrial simulation of the GFDL CM2.1 model (Wittenberg et al. 2006). While the SODA record is too short after 2000 for changes in stability to be disentangled from interannual fluctuations (cf. Fig. 3), the GFDL simulation offers the opportunity to study qualitatively similar changes over a longer time record, at least with respect to ENSO modulation and mean stratification (Kug et al. 2010; Choi et al. 2011; Dewitte et al. 2012).

Figure 6a shows the decadal variability and ENSO modulation of the GFDL simulation. The GFDL model simulates an alternation of periods of increased occurrence of CP and EP El Niño events. Following Kug et al. (2010) and Choi et al. (2011), CP and EP events are defined from a $0.5^{\circ} \mathrm{C}$ threshold value of SST anomalies during November-January in areas Niño4m and $\mathrm{Ni}$ ño3m, respectively. These areas are defined by shifting the usual areas of Niño-4 and Niño-3 $20^{\circ}$ westward 


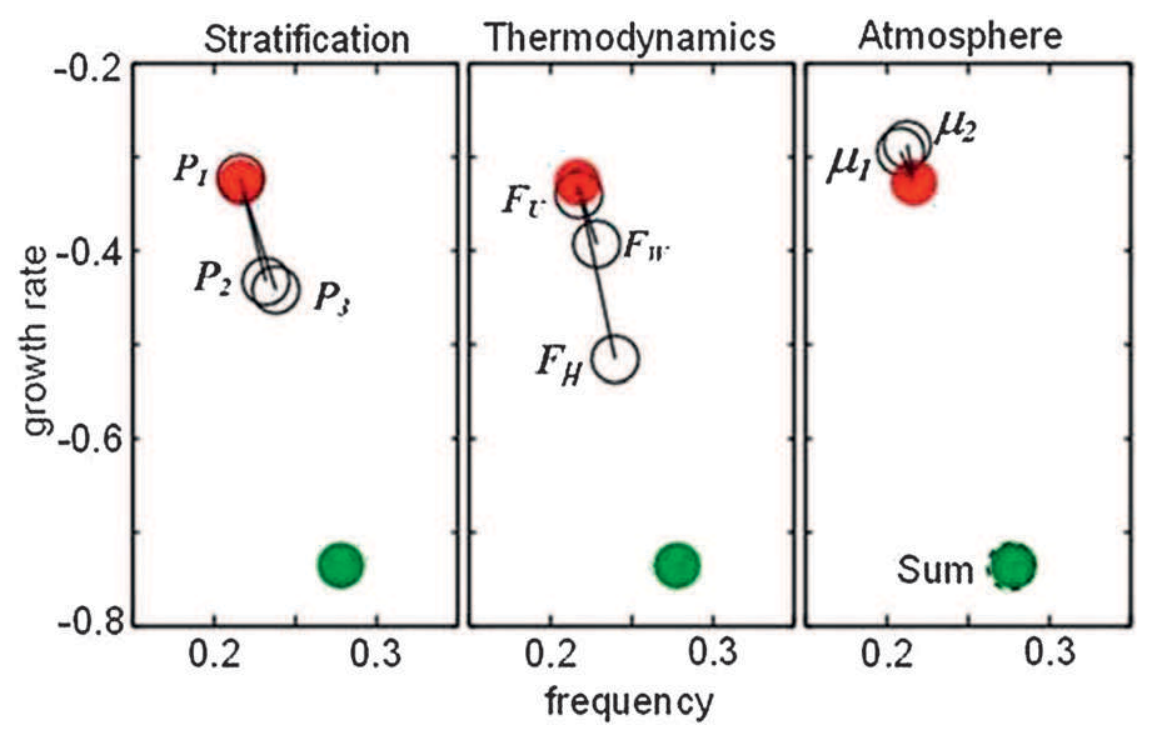

FIG. 7. Frequency $\left(\mathrm{yr}^{-1}\right)$ and growth rate $\left(\mathrm{yr}^{-1}\right)$ of the TD model-simulated ENSO mode, with parameters tuned from the warm (green) or cold mean state (red) of the GFDL CM2.1 simulation. The open black circles are obtained by individually tuning each parameter from the cold mean state with other parameters tuned from the warm mean state. The sum point (black open dashed circle) sums all stability changes from individual parameter changes.

because of the westward shift of variability in the GFDL simulation. Figure 6 a shows that on decadal time scales the occurrence of $\mathrm{CP}$ events (thin green line) is anticorrelated $(r=-0.6)$ with that of EP events (thin red line) and is further correlated $(r=0.7)$ with variations of central Pacific-mean SST (black line). Similarly to Choi et al. (2011), we define a warm (cold) mean state that is averaged over all period intervals where the deviation of the central Pacific-mean SST is superior (inferior) to $0.5^{\circ} \mathrm{C}\left(-0.5^{\circ} \mathrm{C}\right)$ as compared to the full record. This threshold value is lower than $\pm 1^{\circ} \mathrm{C}$ in Choi et al. (2011) because here the simulation is shorter (300 instead of 500 years). The period intervals used for averaging are shown in Fig. 6a for the warm mean state (thick green horizontal lines, top) and the cold mean state (thick red horizontal lines, bottom). Figure $6 \mathrm{~b}$ shows temperature differences between the defined warm and cold states. Interestingly, it shows a strengthening of the equatorial Pacific warm pool similar to the one observed after 2000 on the shorter SODA record (cf. Fig. 1). In this sense, the transition from the cold (with higher occurrence of EP events) to the warm state (with higher occurrence of CP events) in the GFDL simulation is qualitatively similar to the transition after 2000 in the SODA record.

The TD model's parameters are tuned from either the warm or cold mean state of the GFDL simulation. We use a similar method to that in the previous section, first computing a reference state from the full GFDL record and then tuning a reduced set of parameters. This corresponds to a modified scaling of model parameters from the cold to warm mean state, on $P_{1}(+1 \%), P_{2}$ (-9\%), and $P_{3}(-25 \%)$ for upper-ocean stratification; $F_{U}(+4 \%), F_{H}(-7 \%)$, and $F_{W}(+4 \%)$ for mixed layer thermodynamics; and $\mu_{1}(+5 \%)$ and $\mu_{2}(+2 \%)$ for the statistical atmosphere. In addition, because in the GFDL simulation the variability is shifted westward by around $20^{\circ}$, averages are shifted accordingly to compute variations in $P_{n}, F_{H}, F_{U}$, and $F_{W}$. Changes in mean state parameters are qualitatively consistent with Choi et al. (2011), where the warm mean state is associated with a reduced thermocline feedback (i.e., $F_{H}$ ), an enhanced damping feedback caused by enhanced easterlies (i.e., $F_{W}$ ), and a slightly increased atmospheric response to SST anomalies (i.e., $\mu_{1}$ and $\mu_{2}$ ).

Changes in stability are shown in Fig. 7, where the TD model is tuned from either the warm (red) or cold mean state (green) of the GFDL simulation. The TD model simulates a more stable and high-frequency ENSO mode for the warm mean state. As for the climate shift of the 1970s in the SODA record (Fig. 4), changes in stability are mostly controlled by the thermocline feedback (i.e., $F_{H}$ ) and stratification parameters (i.e., $P_{2}$ and $P_{3}$ ). Again, a main conclusion is that the influence of stratification parameters (i.e., $P_{2}$ and $P_{3}$ ) on ENSO stability is comparable in magnitude to that of mixed layer thermodynamics and 
(a) Ensemble Mean

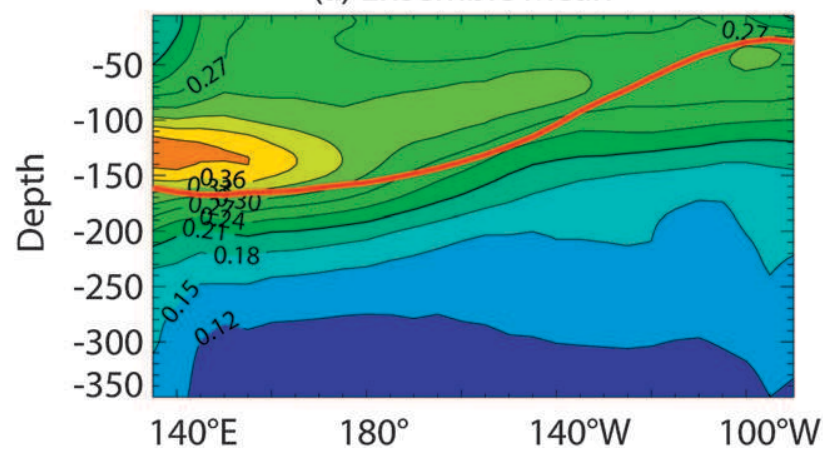

(b) Ensemble RMS

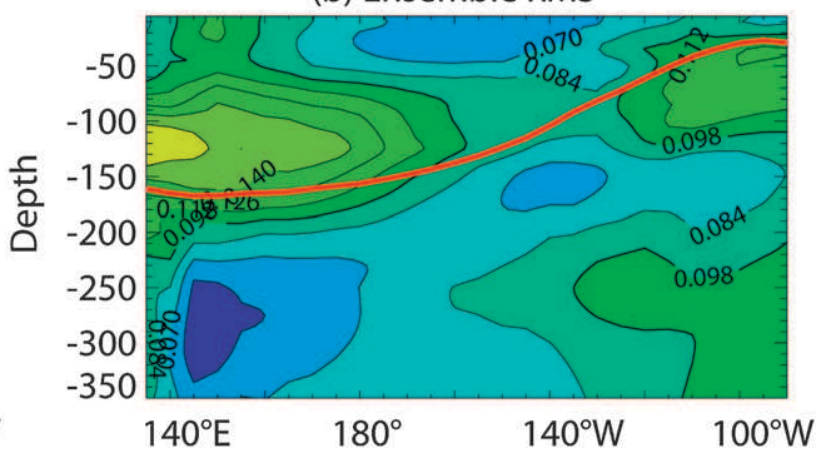

FIG. 8. (a) Ensemble mean and (b) dispersion, among the panel of 15 CGCM simulations, of the mean state temperature variability $\left({ }^{\circ} \mathrm{C}\right.$; defined as std dev of the 10-yr running mean). Results are shown at the equator as a function of longitude and depth. The ensemble-mean thermocline depth is superimposed (red thick line).

atmosphere parameters. This also extends results from Choi et al. (2011) where linear stability is not assessed.

As for the climate shift of the 1970s (cf. Fig. 5), the TD model here simulates a spatially fixed recharge/discharge process with maximal SST anomalies in the eastern Pacific, which is representative of EP events (not shown). For a warmer mean state (with decreased stratification) this eigenmode is more damped, which is qualitatively consistent with the decreased occurrence of EP events. There is, however, no leading eigenmode associated with $\mathrm{CP}$ events, at least with maximal SST anomalies in the central Pacific. Indeed, to our knowledge there are at this time no studies of reduced models such as the TD model that clearly identify an eigenmode representative of $\mathrm{CP}$ events. This limitation will be discussed in the concluding section of this article.

The relation between ENSO modulation and mean stratification is further addressed in a panel of preindustrial simulations of various CGCMs from the CMIP3 archive (see section 2b). Like GFDL CM2.1 (included in the panel), these models simulate their own mean state with a refined representation of the ocean's vertical structure allowed by their relatively high vertical resolution. Figure 8 shows the mean state temperature variability among the ensemble of model simulations. According to the ensemble mean in Fig. 8a, it appears that mean state variability is most pronounced just above the equatorial thermocline and from the central to western Pacific, which is similar to the previously described mean state changes of the SODA reanalysis (cf. Fig. 1) and the GFDL CM2.1 simulation (cf. Fig. 7). The ensemble dispersion in Fig. 8b further shows that this region is also where CGCMs mostly disagree on the intensity of mean state variability. Those considerations may be extended to the $P_{n}$ coefficients, whose mean state variability shows a significant ensemble mean $(1.0 \%, 3.2 \%$, and $6.9 \%$ with respect to the nominal value for $P_{1}, P_{2}$, and $P_{3}$, respectively) and ensemble dispersion $(0.3 \%, 1.5 \%$, and $3.4 \%$ with respect to the nominal value for $P_{1}, P_{2}$, and $P_{3}$, respectively).

We also consider, in the panel of CGCM simulations, the relation between the decadal variability of mean stratification and the simulated ENSO modulation. Table 1 shows correlations between the variations in the $P_{n}$ coefficients and in the index N3Var of ENSO modulation (defined here as the running standard deviation of Niño-3 SST anomalies). Interestingly, various models (in upper rows of Table 1) show a significant correlation $(r>0.6)$ for both $P_{2}$ and $P_{3}$. Meanwhile, variations in $P_{1}$ are rather weakly anticorrelated with ENSO modulation, as is also the case for the mean thermocline depth of the central Pacific. Nevertheless, results from SODA and GFDL CM2.1 suggest that $P_{2}$ and $P_{3}$ have the greatest influence on ENSO stability. In all models the index N4Var (for Niño-4 SST anomalies) is strongly correlated with N3Var $(r>0.7)$, which therefore does not permit a clear separation between periods of increased occurrence of EP or CP events in those relatively short records $(\sim 150 \mathrm{yr})$. However, it is interesting to note that CNRM CM3, GFDL CM2.1, and INM-CM3.0 (but not HadGCM.1) are among the models showing the greatest correlations between stratification changes and ENSO modulation, while they are in the meantime the ones where the relative intensity of $\mathrm{CP}$ and EP events is best represented (Yu and Kim 2010). In conclusion, the decadal variability of mean stratification in the central Pacific (i.e., the $P_{n}$ coefficients) appears to be a robust feature of the panel of CGCM simulations, which is significantly correlated to ENSO modulation in some models, although there are significant discrepancies among models with respect to the intensity of such variability. 


\section{Summary and discussion}

A recharge/discharge conceptual model has been used to document changes in the mean state and stratification of the equatorial Pacific as well as the associated changes in ENSO stability. This relation has been considered for the climate shift of the 1970s in the SODA reanalysis as well as in a preindustrial simulation of GFDL CM2.1 showing stratification changes similar to the ones after 2000. Considerations on the relation between stratification changes and ENSO modulation were also extended to a panel of other preindustrial simulations of coupled global circulation models.

Sensitivity tests have been considered for the relative influence of various mean state parameters onto ENSO stability rather than for one type of parameter alone [see also Jin et al. (2006); Kim and Jin (2011)]. As compared to previous sensitivity studies, the conceptual model used here (Thual et al. 2011) also considers dynamics of the gravest baroclinic modes of the continuously stratified ocean rather than two-layer shallow-water dynamics, which accounts for peculiarities of the mean thermocline structure (thickness, intensity, and overall shape) and their changes. Results from the SODA reanalysis and the GFDL simulation confirm the significant sensitivity of ENSO stability to decadal changes in the ocean stratification parameters (i.e., $P_{n}$ ) that control the efficiency of transfer of work from wind stress forcing to ocean motion. In addition, such sensitivity is comparable in magnitude to the one of usual thermodynamic and atmospheric parameters. This also complements previous studies where the sensitivity to stratification has been assessed through direct numerical simulation (Moon et al. 2004; Dewitte et al. 2007, 2009).

The evidenced sensitivity is, however, limited to the model representation of ENSO dynamics, and the ways that mean state feedbacks are estimated. The reduced model is indeed too simple to account for all the many features of ENSO modulation. It successfully simulates a dominant oscillating eigenmode, qualitatively representative of ENSO oscillations, where the main process is a recharge/discharge on the equatorial thermocline (Jin 1997a,b). However, because of its statistical parameterization of the atmospheric response, the model favors standing SST modes rather than propagating SST modes like in the observed record (An et al. 2006). For this reason, its sensitivity to ocean stratification is mostly on the frequency and growth rate of the simulated eigenmode. In particular, for a realistic parameter range (e.g., as derived from the GFDL CM2.1 simulation) the reduced model does not simulate an eigenmode representative of CP El Niño events. Indeed, to our knowledge there are at this time no studies of reduced models such as the one proposed here that clearly identify an eigenmode representative of $\mathrm{CP}$ events. Accounting for this may involve additional parameter tunings (e.g., of the thermocline feedback in the central Pacific; cf. Dewitte et al. 2013), or additional mechanisms, including nonlinear ones (Philip and van Oldenborgh 2009). Kug et al. (2010), for example, has suggested from composites of the GFDL CM2.1 simulation that CP El Niño might be representative of a damped westward propagating mode of SST anomalies, involving mainly atmospheric processes. In addition, we may wonder if CP El Niño can be accounted for by either modifications of the leading eigenmode (Fedorov and Philander 2001) or rather as a secondary eigenmode competing in terms of growth rate with the leading eigenmode (Bejarano and Jin 2008).

One general and critical challenge for reduced ENSO models is to account, at the same time, for both a more refined representation of the observed processes as well as a more synthetic view at the most influential ones. This suggests two complementary perspectives for future works on sensitivity to ocean stratification. First, we may further refine the representation of ocean stratification in the reduced model, for example by considering the contribution of higher ocean baroclinic modes (TD2011) or by accounting for zonal stratification changes (Busalacchi and Cane 1988). Second, it would be interesting to provide a more synthetic view at the sensitivity to ocean stratification in order to ease CGCM intercomparison. For example, the linear stability of the proposed reduced model shares many characteristics with the Bjerknes index (Jin et al. 2006; Kim and Jin 2011), such as a recharge/ discharge process with fixed spatial structure, as well as a growth rate of the simulated ENSO depending linearly on the contribution of various mean state feedbacks. Therefore it may be interesting to assess sensitivity to ocean stratification within this synthetic and versatile framework, although the consideration of the gravest ocean baroclinic modes would render the derivation of the Bjerknes index more complex.

In conclusion, our results call for consideration of stratification changes in order to interpret more aspects of the modulation and diversity of ENSO in observations (e.g., for the climate shift of the 1970s) and in CGCMs that have a refined representation of the ocean's vertical structure. Notably, the diversity of ENSO variability in CGCMs goes along with significant model dispersion in terms of mean stratification and its low-frequency variability (see, e.g., Fig. 8 and Table 1 for the CMIP3 archive). This is also likely to have implications for the CGCM responses to global warming (Guilyardi et al. 2009) where mean stratification is expected to increase significantly. It is proposed here to include metrics related to ocean stratification for the evaluation of the CGCMs. The 
approach and results proposed in this study may provide material for elaborating such metrics, which is planned for future work.

Acknowledgments. The authors would like to thank the two anonymous reviewers for their constructive comments that helped improve the original manuscript, as well as Professor S.-W. Yeh and B. Meyssignac for fruitful discussions. S. Thual has been supported by Centre National de la Recherche Scientifique (CNRS) and Conseil Régional Midi-Pyrénées under Contract 022009. This work is a contribution to a Science and Technology Amicable Research (STAR) program funded by the CNRS and the National Research Foundation of Korea (NRF-2009 C1AAA001-2009-0093042). The authors also acknowledge support from Centre National d'Etudes Spatiales (CNES) through the Modokalt OSTST project.

\section{REFERENCES}

An, S.-I., and F.-F. Jin, 2000: An eigen analysis of the interdecadal changes in the structure and frequency of ENSO mode. Geophys. Res. Lett., 27, 2573-2576.

- , and B. Wang, 2000: Interdecadal change of the structure of the ENSO mode and its impact on the ENSO frequency. J. Climate, 13, 2044-2055.

_ , and F.-F. Jin, 2001: Collective role of thermocline and zonal advective feedbacks in the ENSO mode. J. Climate, 14, 3421-3432.

- Z. Ye, and W. W. Hsieh, 2006: Changes in the leading ENSO modes associated with the late 1970s climate shift: Role of surface zonal current. Geophys. Res. Lett., 33, L14609, doi:10.1029/2006GL026604

_, J.-S. Kug, Y.-G. Ham, and I.-S. Kang, 2008: Successive modulation of ENSO to the future greenhouse warming. J. Climate, 21, 3-21.

Ashok, K., S. K. Behera, S. A. Rao, H. Weng, and T. Yamagata, 2007: El Niño Modoki and its possible teleconnection. J. Geophys. Res., 112, C11007, doi:10.1029/2006JC003798.

Bejarano, L., and F.-F. Jin, 2008: Coexistence of equatorial coupled modes of ENSO. J. Climate, 21, 3051-3067.

Belmadani, A., B. Dewitte, and S.-I. An, 2010: ENSO feedbacks and associated time scales of variability in a multimodel ensemble. J. Climate, 23, 3181-3204.

Busalacchi, A. J., and M. A. Cane, 1988: The effect of varying stratification on low-frequency equatorial motions. J. Phys. Oceanogr., 18, 801-812.

Carton, J. A., and B. S. Giese, 2008: A reanalysis of ocean climate using Simple Ocean Data Assimilation (SODA). Mon. Wea. Rev., 136, 2999-3017.

Choi, J., S.-I. An, J.-S. Kug, and S.-W. Yeh, 2011: The role of mean state on changes in El Niño's flavor. Climate Dyn., 37, 12051215, doi:10.1007/s00382-010-0912-1.

Collins, M., and Coauthors, 2010: The impact of global warming on the tropical Pacific Ocean and El Niño. Nat. Geosci., 3, 391397, doi:10.1038/ngeo868

Dewitte, B., 2000: Sensitivity of an intermediate ocean-atmosphere coupled model of the tropical Pacific to its oceanic vertical structure. J. Climate, 13, 2363-2388.
S.-W. Yeh, B.-K. Moon, C. Cibot, and L. Terray, 2007: Rectification of ENSO variability by interdecadal changes in the equatorial background mean state in a CGCM simulation. J. Climate, 20, 2002-2021.

-, S. Thual, S.-W. Yeh, S.-I. An, B.-K. Moon, and B. S. Giese, 2009: Low-frequency variability of temperature in the vicinity of the equatorial Pacific thermocline in SODA: Role of equatorial wave dynamics and ENSO asymmetry. J. Climate, 22, 5783-5795.

, J. Choi, S.-I. An, and S. Thual, 2012: Vertical structure variability and equatorial waves during central Pacific and eastern Pacific El Niños in a coupled general circulation model. Climate Dyn., 38, 2275-2289.

- , S.-W. Yeh, and S. Thual, 2013: Reinterpreting the thermocline feedback in the western-central equatorial Pacific and its relationship with the ENSO modulation. Climate Dyn., doi:10.1007/s00382-012-1504-z, in press.

Fedorov, A. V., and S. G. Philander, 2001: A stability analysis of tropical ocean-atmosphere interactions: Bridging measurements and theory for El Niño. J. Climate, 14, 3086-3101.

Guilyardi, E., A. Wittenberg, A. Fedorov, M. Collins, C. Wang, A. Capotondi, G. J. van Oldenborgh, and T. Stockdale, 2009: Understanding El Niño in ocean-atmosphere general circulation models: Progress and challenges. Bull. Amer. Meteor. Soc., 90, 325-340.

Jin, F.-F., 1997a: An equatorial ocean recharge paradigm for ENSO. Part I: Conceptual model. J. Atmos. Sci., 54, 811-829. 1997b: An equatorial ocean recharge paradigm for ENSO. Part II: A stripped-down coupled model. J. Atmos. Sci., 54, 830-847.

_, 2001: Low-frequency modes of tropical ocean dynamics. J. Climate, 14, 3874-3881.

_ J. D. Neelin, and M. Ghil, 1994: El Nino on the devil's staircase: Annual subharmonic steps to chaos. Science, 264, 70-72. S. T. Kim, and L. Bejarano, 2006: A coupled-stability index for ENSO. Geophys. Res. Lett., 33, L23708, doi:10.1029/ 2006GL027221.

Kim, S. T., and F.-F. Jin, 2011: An ENSO stability analysis. Part II: Results from the twentieth and twenty-first century simulations of the CMIP3 models. Climate Dyn., 36, 1609-1627, doi:10.1007/s00382-010-0872-5.

Kirtman, B. P., and P. S. Schopf, 1998: Decadal variability in ENSO predictability and prediction. J. Climate, 11, 2804-2822.

Kug, J.-S., F.-F. Jin, and S.-I. An, 2009: Two types of El Niño events: Cold tongue El Niño and warm pool El Niño. J. Climate, 22, 1499-1515.

_ J. Choi, S.-I. An, F.-F. Jin, and A. T. Wittenberg, 2010: Warm pool and cold tongue El Nino events as simulated by the GFDL 2.1 coupled GCM. J. Climate, 23, 1226-1239.

Lee, T., and M. J. McPhaden, 2010: Increasing intensity of El Niño in the central-equatorial Pacific. Geophys. Res. Lett., 37, L14603, doi:10.1029/2010GL044007.

Lin, J.-L., 2007: Interdecadal variability of ENSO in 21 IPCC AR4 coupled GCMs. Geophys. Res. Lett., 34, L12702, doi:10.1029/ 2006 GL028937.

McPhaden, M. J., 2012: A 21st century shift in the relationship between ENSO SST and warm water volume anomalies. Geophys. Res. Lett., L09706, doi:10.1029/2012GL051826.

Moon, B.-K., S.-W. Yeh, B. Dewitte, J.-G. Jhun, I.-S. Kang, and B. P. Kirtman, 2004: Vertical structure variability in the equatorial Pacific before and after the Pacific climate shift of the 1970s. Geophys. Res. Lett., 31, L03203, doi:10.1029/ 2003GL018829. 
Neelin, J. D., D. S. Battisti, A. C. Hirst, F.-F. Jin, Y. Wakata, T. Yamagata, and S. E. Zebiak, 1998: ENSO theory. J. Geophys. Res., 103, 14 261-14 290.

Philander, S. G., and A. Fedorov, 2003: Is El Niño sporadic or cyclic? Annu. Rev. Earth Planet. Sci., 31, 579-594.

Philip, S., and G. J. van Oldenborgh, 2009: Significant atmospheric nonlinearities in the ENSO cycle. J. Climate, 22, 4014-4028.

Rodgers, K. B., P. Friederichs, and M. Latif, 2004: Tropical Pacific decadal variability and its relation to decadal modulations of ENSO. J. Climate, 17, 3761-3774.

Seager, R., A. R. Karspeck, M. A. Cane, Y. Kushnir, A. Giannini, A. Kaplan, B. Kerman, and J. Velez, 2004: Predicting Pacific decadal variability. Earth's Climate: The Ocean-Atmosphere Interaction, Geophys. Monogr., Vol. 147, Amer. Geophys. Union, 105-120.
Sun, D.-Z., and T. Zhang, 2006: A regulatory effect of ENSO on the time-mean thermal stratification of the equatorial upper ocean. Geophys. Res. Lett., 33, L07710, doi:10.1029/2005GL025296.

Thual, S., B. Dewitte, S.-I. An, and N. Ayoub, 2011: Sensitivity of ENSO to stratification in a recharge-discharge conceptual model. J. Climate, 24, 4332-4349.

Wang, B., and Y. Wang, 1996: Temporal structure of the Southern Oscillation as revealed by waveform and wavelet analysis. J. Climate, 9, 1586-1598.

Wittenberg, A. T., A. Rosati, N.-C. Lau, and J. J. Ploshay, 2006: GFDL's CM2 global coupled climate models. Part III: Tropical Pacific climate and ENSO. J. Climate, 19, 698-722.

Yu, J.-Y., and S. T. Kim, 2010: Identification of central-Pacific and eastern-Pacific types of ENSO in CMIP3 models. Geophys. Res. Lett., 37, L15705, doi:10.1029/2010GL044082. 